Research Paper

\title{
Detection Technology of Road Marks Utilizing Combination of Partial Templates
}

\author{
Satoshi Nakamura ${ }^{1)}$ Takuma Ito ${ }^{1)}$ Toshiki Kinoshita ${ }^{2)}$ Minoru Kamata ${ }^{1)}$ \\ 1) The University of Tokyo \\ 5-1-5 Kashiwanoha, Kashiwa, Chiba,277-8589, Japan (E-mail: nakamura.satoshi@atl.k.u-tokyo.ac.jp) \\ 2) Toyota Motor Corporation \\ 1200 Mishuku, Susono, Shizuoka, 410-1193, Japan
}

Received on October 12, 2017

\begin{abstract}
To detect road marks as the references of localization for automated driving technologies, we developed a detection system which is robust against disturbances such as shadows, degradation, occlusions, and changes of road surface appearances due to different weather conditions. For the accurate distance estimation, our system used the "camera-down system"; furthermore, for making the most of it, our system used the combinations of multi partial templates which focused on different parts of road marks. From evaluations of actual driving data on a prefectural road in several weather conditions, we confirmed that our proposed system has the practical performances for correctly detecting road marks including disturbed ones.
\end{abstract}

KEY WORDS: safety, intelligent vehicle, road environment, image processing / road mark, partial template [C1]

\section{Introduction}

Recently, many researchers are developing localization technologies for advanced driver assistance systems (ADAS), such as speed control system ${ }^{(1)(2)}$ based on digital maps which provide the information about the upcoming road. Some of existing researchers focused on simultaneous localization and mapping (SLAM) methods for localization of intelligent vehicles. For example, some research groups ${ }^{(3)(4)(5)}$ proposed localization systems based on SLAM methods and they showed high precision and robustness in the real road environments. However, they have problems of high-cost sensors and large amounts of digital data for the popular use of automated driving technologies in rural areas. Therefore, our research group ${ }^{(6)}$ developed the automated driving system based on the lean map platform; further, we confirmed the feasibility of the concept through the autonomous driving experiment on a test course using the prototype of proposed system without high-cost sensors or large amounts of data. Although previously proposed system utilized elements of traffic environments as the references of longitudinal localization (hereinafter, these elements are called "landmarks"), the prototype used only stop lines and guardrails as landmarks, which existed on the test course; thus, we need to discuss the use of other landmarks on public roads. Therefore, in this paper, we will discuss the requirements of landmarks on public roads and select the appropriate elements of traffic environments for landmarks. Then we will design a robust detection system of the landmarks which are selected for localization on public roads. To be more precise, we develop the detection system with a procedure as follows:

1. Discuss the requirements of landmarks on public roads.

2. Select the appropriate elements of traffic environments for landmarks.
3. Design the detection system utilizing the lean map information.

4. Evaluate the detection performance of the proposed system on public roads.

\section{Detection System of Road Marks based on Partial Templates}

\subsection{Requirements of Landmarks for Localization}

As discussed in our previous study ${ }^{(6)}$, the localization for the automated driving system should be precise to some extent. To be more precise, we aimed to fulfill the precision of less than $0.5 \mathrm{~m}$ as the first step. Further, it is desirable that the system conducts the localization based on landmarks frequently at a certain level on rural public roads. Regarding these discussions, we organized the requirements of landmarks for localization as follows:

- Longitudinal positions of landmarks can be uniquely determined.

- Landmarks are widely distributed at a certain interval on public roads in rural areas.

- Landmarks can be detected by simple vehicular sensors.

Although the prototype in the previous report ${ }^{(6)}$ focused on guardrails at T-junctions and stop lines as the landmarks, we need to focus on additional landmarks for more frequent localization on public roads. Since there are various types of elements which compose traffic environments in public roads, a number of elements fulfill the abovementioned requirements of landmarks for localization. In this study, we focus on speed limit marks and diamond-shaped marks. In Japan, the diamond-shaped marks alert drivers to the approach of unsignalized crosswalks whereas they indicate the high-occupancy vehicle (HOV) lanes in several countries. In this study, we call these diamond-shaped marks 
"diamarks" from this point forward. We selected these marks from the three viewpoints as follows:

- The size of marks is standardized.

- The shape of marks has characteristics.

- The marks have distinctive features so that detectors can easily distinguish them from surrounding elements.

Although there are several types of speed limit marks, we selected the $40 \mathrm{~km} / \mathrm{h}$ limit marks as the first step, which are common on public roads. In addition, the Ministry of Land, Infrastructure, Transport and Tourism (MLIT) of Japan standardizes the size of the $40 \mathrm{~km} / \mathrm{h}$ limit marks and the diamarks. Figs. 1 and 2 show the standard size of them, respectively.

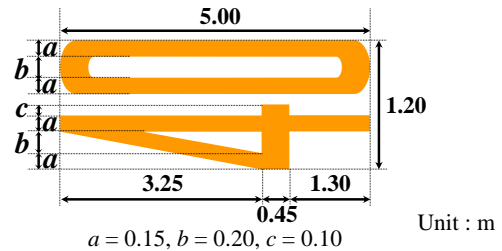

Fig. 1 Standard size of the $40 \mathrm{~km} / \mathrm{h}$ limit marks.

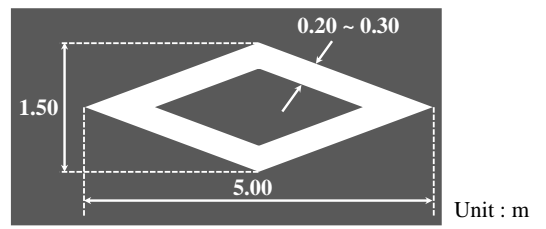

Fig. 2 Standard size of the diamarks.

\subsection{Limitations of Existing Technologies for Our Purpose}

In accordance with the recent development of ADAS aiming the reduction of traffic accidents, road marks are focused as indicators of road environments such as a speed limit in an area, an approach of crosswalks, and so on. To grasp the road environments via road marks, many existing studies have developed detection technologies of road marks. However, the existing technologies still have challenges about the robustness against the disturbances such as degradation, shadows, occlusions, and so on. To improve the robustness of the road mark detection, Noda et al. ${ }^{(7)}$ proposed the detection system by a generative learning method, which is robust against the appearance variation. Further, Yoneyama et al. ${ }^{(8)}$ reported the detection method using hidden Markov model (HMM) and showed that the system detects degraded road marks. In addition, Ishida et al. ${ }^{(9)}$ showed that the detection technology based on tensor voting can detect degraded road marks as long as characteristic parts of road marks remain undegraded.

Since the abovementioned existing researches focused on the road marks as the indicators of road environments, their systems need not only the function of detecting the road marks but also the function of identifying their types. On the other hand, since we focus on the road marks as the references for localization, distance estimation is more important than discrimination of road marks. Thus, our system needs only the function of detecting the characteristic regions extracted from the road marks. Therefore, we can design our system to detect the characteristic regions of the road marks with which existing methods can not distinguish the target road marks from similar ones.

\subsection{Detection Strategies based on the Effective Use of Map Data}

Regarding the discussion in the previous section, since the localization system in our study utilizes the lean map data, the detection system of road marks can obtain the information on their types and their positions in advance. On the assumption that the system is designed to detect not the whole road marks but the characteristic parts of road marks, it has merits for dealing with disturbances as follows:

- For degradation: Since the wear between tires and road surfaces caused the degradation of road marks, there are two regions on them: regions which are easily degraded and regions which are hardly degraded. Thus, the system can detect road marks by finding the latter regions. Fig. 3 shows the conceptual schematic of the strategy for detecting degraded road marks. As is observable in the figure, even if the both side regions of a road mark are degraded, we can estimate the whole shape of the road mark by detecting other characteristic regions such as the region framed by magenta lines.

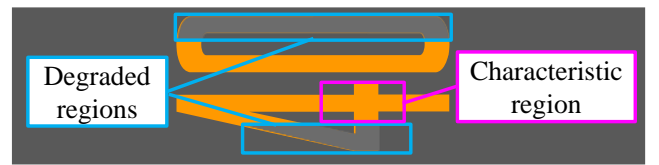

Fig. 3 Conceptual schematic of the strategy for detecting degraded road marks.

- For shadow: The system can detect characteristic regions unless the brightness changes dramatically in the regions. In other words, on the conditions that characteristic regions of road marks are "completely covered with shadows" or "not covered with shadows at all", we can detect the road marks by those characteristic regions. Fig. 4 shows the conceptual schematic of the strategy for detecting road marks covered with shadows. As is shown in the figure, even if the upper half region of a road mark is covered with a shadow, we can estimate the whole shape of the road mark by detecting the abovementioned regions such as the regions framed by magenta lines.

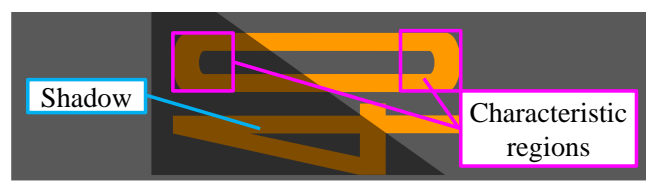

Fig. 4 Conceptual schematic of the strategy for detecting road marks covered with shadows.

- For occlusion: On the condition that one or more characteristic regions are not occluded, the system can detect the road marks by those regions. Fig. 5 shows the conceptual schematic of the strategy for detecting road marks with occlusions. As is observable in the figure, even if the lower half region of a road mark is occluded by the following vehicle, we can estimate the whole shape of the road mark by detecting other characteristic regions such as the region framed by magenta lines. 


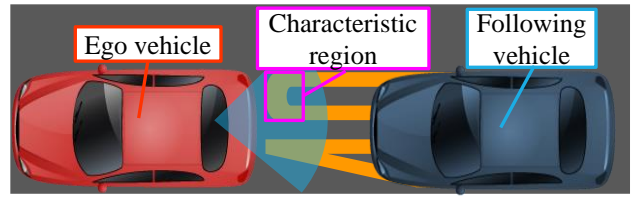

Fig. 5 Conceptual schematic of the strategy for detecting road marks with occlusions.

\subsection{Detection by Rear View Camera}

As we discussed in section 2.1., the sensors for our detection system need to be simple enough to realize easy popularization in the near future. Therefore, intending to utilize popular parking assist cameras, we equipped the rear window of the experimental vehicle with a USB camera (hereinafter, this USB camera is called "rear view camera"). Table 1 shows the specifications of the rear view camera.

Table 1 Specifications of the rear view camera.

\begin{tabular}{|c|c|}
\hline Model & Logicool HD Pro Webcam C920r \\
\hline Angle of view & 77 degrees \\
\hline Resolution & 640 x 480 pixels \\
\hline Frame rate & $30 \mathrm{fps}$ \\
\hline
\end{tabular}

In general, front view cameras can not take road surface images just in front of the vehicle due to the engine hood. On the contrary, the rear view camera can take road surface images just behind the vehicle. This feature of the rear view camera associated with the coverage field has two merits for detecting road marks.

First, the system can grasp accurately the distance between the ego vehicle and the detected road mark. Since the goal of our detection system is localization, the system needs to not only detect road marks, but also calculate the accurate distance from the ego vehicle to them. Considering the accurate calculation of the abovementioned distance, the changes in the depression angles of cameras, which are caused by postural changes of the ego vehicle such as nosedives, affect the calculation considerably. Fig. 6 shows the conceptual schematic of the changes in coverage fields of cameras caused by postural changes. The red area indicates the coverage field of the front view camera, which we call as "camera-up system" contrastively, whereas the blue area indicates that of the rear view camera, which we call as "cameradown system" contrastively. When the camera angles change due to the postural changes of the vehicle, the coverage fields of the front view camera and the rear view camera change into the areas framed by red dotted lines and blue dotted lines, respectively. At this point, the deviation on the road surface of the rear view camera $\left(D_{r}\right)$ is smaller than that of the front view camera $\left(D_{f}\right)$. Therefore, using the rear view camera, whose depression angle is larger than that of the front view camera, we suppose that the system can calculate the distance robustly against the postural changes of the vehicle.

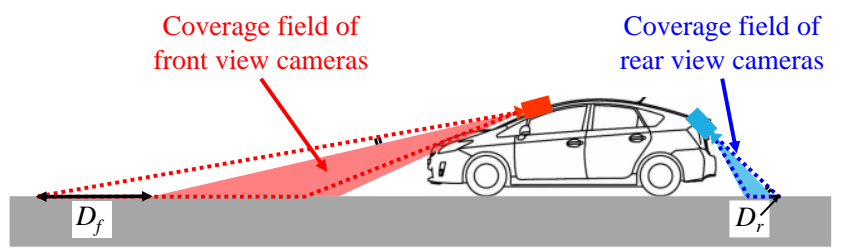

Fig. 6 Conceptual schematic of the changes in coverage fields of cameras caused by postural changes.

Second, the system can diminish the effect of occlusions by other vehicles. Since the road surface images obtained via the rear view cameras cover nearer ranges than those obtained via the front view cameras cover, the probability that other vehicles occlude road marks becomes low.

Although abovementioned two merits become more effective in accordance with the increase in the depression angle of the rear view camera, the coverage field of the camera becomes smaller as the camera looks more downward. In short, the case that the whole shape of a road mark exceeds the coverage field can occur. However, our proposal, which focuses on the characteristic regions of road marks, has the possibility of detecting them successfully even if the whole road marks are not within the coverage field of the camera. In short, focusing on characteristic regions of road marks can enhance the advantages of the rear view camera.

\subsection{Prototype Implementation}

Based on the proposal for a robust detection system of road marks discussed in the sections 2.3. and 2.4., we implemented a prototype of the detection system focusing on the characteristic regions of road marks and the rear view camera.

\subsubsection{Overview}

Fig. 7 shows the schematic of the rear view camera on the experimental vehicle. We equipped the vehicle with the rear view camera, whose depression angle is 40 degrees.

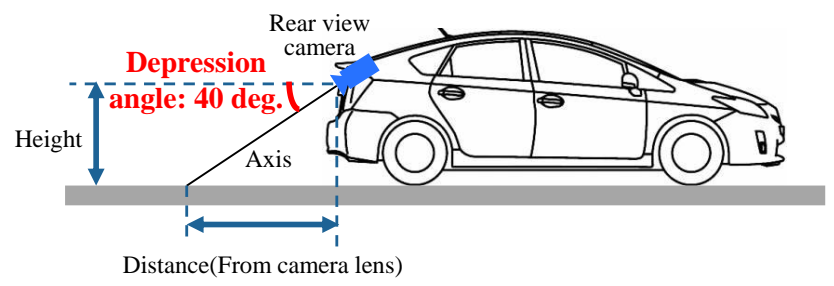

Fig. 7 Schematic of rear view camera on the experimental vehicle.

Fig. 8 shows the system configuration diagram of the detection system developed in this research. To implement this system, we used ROS, which is a middleware for development of robots. First, the ADAS Horizon Provider ${ }^{(6)}$, which provides the information of map, receives the GPS information and estimates the position of the ego vehicle roughly. Subsequently, ADAS Horizon Provider sends the position information and the type information of the approaching road mark to the road mark detector. Eventually, based on the abovementioned information about the approaching road mark and the images captured by the rear view camera, the road mark detector starts operating at $5 \mathrm{~m}$ in front of the road mark and keeps operating during next $50 \mathrm{~m}$; thus, 
we can reduce false detections by limiting the detection range based on the map information.

\begin{tabular}{|c|c|c|c|c|}
\hline \multirow{3}{*}{ GPS } & \multirow{3}{*}{$\begin{array}{l}\text { Vehicle } \\
\text { position }\end{array}$} & \multicolumn{3}{|c|}{$\begin{array}{c}\text { Information of } \\
\text { the approaching road mark }\end{array}$} \\
\hline & & ADAS Horizon & (Position, Type) & Road mark \\
\hline & & Provider & & detector \\
\hline Rear vie & & & amera image & \\
\hline
\end{tabular}

Fig. 8 System configuration diagram.

Furthermore, Fig. 9 shows the schematic of the detection flow in the road mark detector. The details are described in the following sections.

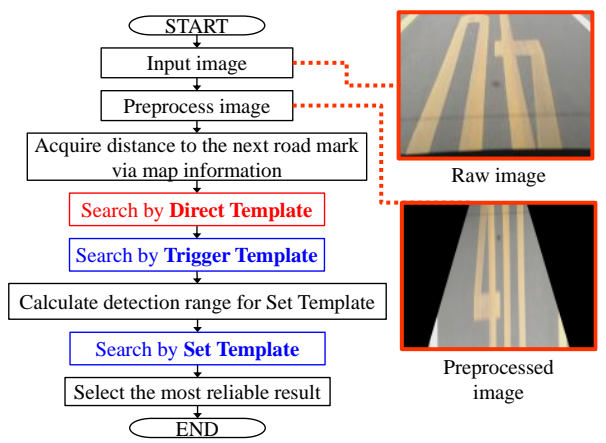

Fig. 9 Schematic of detection flow.

\subsubsection{Detecting Characteristic Regions of Road Marks}

In our detection system, we implemented a two-step image processing function. To implement this function, we used OpenCV, which is an open source computer vision library.

The first step is preprocessing of captured images. First, the detector undistorts the camera images, and then generates birds' eye view images by the projection transformation. Since the size of road marks in the transformed images is constant regardless of the positions where they are in the images, the following template matching process becomes simple. In addition, the system reduces the size of the abovementioned transformed images so as to realize the real-time detection.

The second step is detecting characteristic regions of road marks based on the template matching method. The road marks selected as the landmarks for localization have the standardized sizes and shapes. Moreover, the direction in which vehicles pass over road marks is mostly fixed. Therefore, since we suppose that the detector does not need to consider the variations in neither size nor direction of road marks, we used the "template-based template matching method". The template-based template matching methods have two approaches: the approach based on edge patterns and the approach based on luminance patterns. Considering the degraded road marks, whose changes in edge patterns are so considerable that detection becomes difficult, we selected the approach by luminance patterns. Moreover, we implemented the method that the detector calculates Zero-mean Normalized Cross Correlation (ZNCC) as an index of similarity between a template image and target regions because this method is relatively robust against changes in brightness. The ZNCC takes a value within the range from -1.0 to +1.0 . Furthermore, as the ZNCC approaches to +1.0 , the target region is similar to the template image. The ZNCC between the template image $(T)$ and the examined image $(I)$ at the pixel position $(\mathrm{x}, \mathrm{y})$ can be written as follows:

$$
\begin{aligned}
& Z N C C(x, y) \\
& =\frac{\sum_{j}^{m} \sum_{i}^{n}\left(I(x+i, y+j)-\mu\left(I_{s}(x, y)\right)\right)(T(i, j)-\mu(T))}{\sqrt{\sum_{j}^{m} \sum_{i}^{n}\left(I(x+i, y+j)-\mu\left(I_{S}(x, y)\right)\right)^{2} \times \sum_{j}^{m} \sum_{i}^{n}(T(i, j)-\mu(T))^{2}}}
\end{aligned}
$$

where $I$ is the image under examination, of size $W * H$ pixels, $T$ is the template image, of size $M * N$ pixels, and $I_{s}(x, y)$ is the subimage, of size $M * N$ pixels, located at $(x, y)$. In addition, $\mu(x, y)$ and $\mu(T)$ are the mean luminance values of $T$ and $I_{s}(x, y)$. For detecting the target from an image frame, the detector calculates $R_{Z N C C}$, which is the maximum value of $\operatorname{ZNCC}(x, y)$ in a frame, as follows:

$$
R_{Z N C C}=\max _{0 \leq x \leq W-M, 0 \leq y \leq H-N} Z N C C(x, y)
$$

We selected multiple regions on road marks as the template images considering the characteristics of luminance patterns and the positions on road marks. We call the abovementioned regions as partial templates from this point forward. Figs. 10 and 11 show the partial templates of the $40 \mathrm{~km} / \mathrm{h}$ limit marks and the diamarks. Hereinafter, each partial template is referred in the simple form such as " $40 \_3$ ". Since the detector can obtain the type information of road marks from the map data, we can use partial templates with which the detector itself can not identify the type of target road marks such as "40_1". To be more precise, although $30 \mathrm{~km} / \mathrm{h}$ limit marks and $50 \mathrm{~km} / \mathrm{h}$ limit marks also have the same region as "40_1", our detection system using map data do not need to distinguish them for localization. This is the important point of the proposed method.

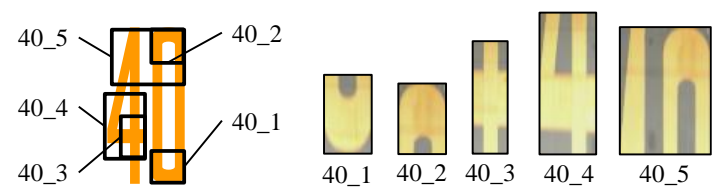

Fig. 10 Partial templates of the $40 \mathrm{~km} / \mathrm{h}$ limit marks.

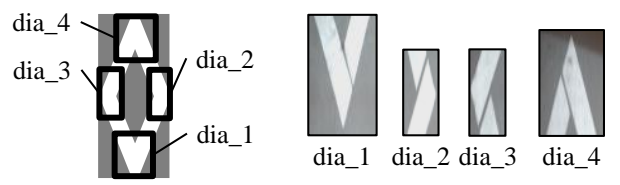

Fig. 11 Partial templates of the diamarks.

\subsubsection{Combinational Use of Partial Templates}

Regarding the multiple partial templates on each road mark, we aimed to enhance the detection performance based on positional relationships between more than one partial templates. To be more precise, considering the sequence of the detection, we aimed to reduce false detections by limiting the detection ranges for partial templates detected in latter parts. In this subsection, we illustrate the method limiting the detection range in longitudinal and lateral directions with the example of the $40 \mathrm{~km} / \mathrm{h}$ limit mark. Regarding the diamarks, we implemented the combinational use of partial templates in a similar method. Figs. 12 and 13 show the 
conceptual schematics of detecting partial templates based on longitudinal and lateral positional relationships, respectively.

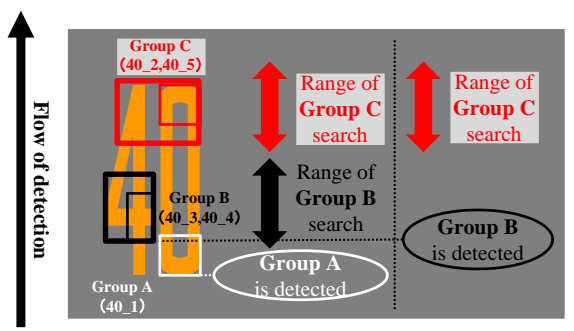

Fig. 12 Conceptual schematic of detecting partial templates based on longitudinal positional relationship.

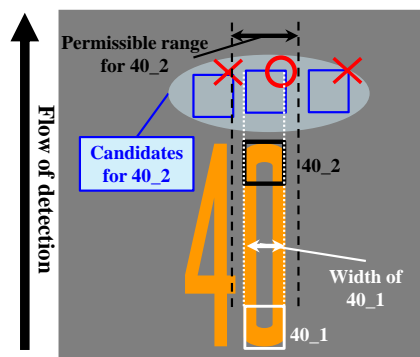

Fig. 13 Conceptual schematic of detecting partial templates based on lateral positional relationship.

Regarding the longitudinal direction, first, we divided the five partial templates into three groups (Group A, B, and C) based on their longitudinal positions. As shown in Fig. 12, when the partial template belonging to the Group A is detected, the detector searches templates belonging to the Group B in the range of the black double-headed arrow, and those belonging to the Group C in the range of the red double-headed arrow. Similarly, when the templates belonging to the Group $\mathrm{B}$ are detected, the detector searches templates belonging to the $\mathrm{C}$ in the range of the red double-headed arrow in Fig. 12.

Regarding the lateral direction, using the detection results of "40_1" and "40_3", the detector determines whether the candidates for latter templates are rejected or accepted. Fig. 13 shows the example that the system evaluates the candidates for " $40 \_2$ " by the detection result of " $40 \_1$ ".

\subsubsection{Determination of Threshold Value for Template Matching}

In this subsection, we discuss the threshold values for RZNCC, which are criteria for detecting the road marks. Considering the combinations of partial templates discussed in the previous subsection, we defined three kinds of roles for partial templates and organized the requirements of the threshold value for each role as follows:

- Direct Template: Once a partial template playing this role is detected, the system determines that the target road mark is found. Thus, we need to set the threshold values so as to fulfill the requirement of Direct Template. To be more precise, non-detection is permissible to some extent, meanwhile false detection is not permissible.

- Trigger Template: When a partial template plays this role, the positive result of the partial template limits the detection ranges for the following detection process. Thus, we need to set the threshold values so as to fulfill the requirement of Trigger Template. To be more precise, false detection is permissible to some extent, meanwhile non-detection is not permissible.

- Set Template: In case that partial templates were detected by templates working as Trigger Templates, the corresponding partial template plays this role in the limited detection range. Once the partial template is detected, the system determines that the target road mark is found. Thus, we need to set the threshold values so as to fulfill the requirement of Set Template. To be more precise, nondetection is permissible to some extent, meanwhile false detection is not permissible.

Regarding the three kinds of roles discussed above, partial templates play more than one roles at a same time in accordance with their positions in the road marks. For example, "40_1", which can be detected in the first step, plays two roles: Direct Template and Trigger Template for "40_2", "40_3", "40_4", and "40_5". In another case, "40_3", which can be detected in the middle step, plays three roles: Direct Template, Trigger Template for "40_2" and "40_5", and Set Template. In addition, "40_2", which can be detected in the last step, plays two roles: Direct Template and Set Template.

To determine the threshold values of partial templates based on the above mentioned requirement of each role, we conducted a preliminary test in the following steps:

1. Under cloudy conditions, the rear view camera took the images of road surface around the $40 \mathrm{~km} / \mathrm{h}$ limit marks and diamarks. Next, we selected 18 of the $40 \mathrm{~km} / \mathrm{h}$ limit marks and 14 of the diamarks, which were not affected by disturbances such as degradation, shadows, and occlusions.

2. We extracted all frames of images within $50 \mathrm{~m}$ from the road marks. Subsequently, if a frame completely included the regions corresponding to the partial templates, we classified the frame into the positive sample. Otherwise, we classified it into the negative sample.

3. Based on the calculated $R_{Z N C C}$ of each partial template in the extracted images, we generated histograms whose bin width is 0.05 for positive and negative samples, respectively. Eventually, we determined the thresholds in accordance with the rules as follows:

- Trigger Template: The threshold is the lower limit value of the bin including the positive sample whose RZNCC is minimum ("First step" in Fig. 14). At this point, if the accumulated composition rate of negative samples which are summed up at all the bins upper than that of "First step" does not exceed 5\%, we lower the bin until the accumulated composition rate of negative samples exceeds 5\%. The threshold is the upper limit value of that bin ("Second step" in Fig. 14).

- Set Template: The threshold is the lower limit value of the lowest bin which includes no negative samples.

- Direct Template: The threshold is the largest value of the thresholds for Set Templates.

Fig. 14 shows the histograms of $R_{Z N C C}$ and the threshold values for "dia_1", whose roles are the Trigger Template and the Direct Template. On the other hand, Fig. 15 shows the histograms of $R_{Z N C C}$ and the threshold values for "dia_3", which plays the roles as the Set Template and the Direct Template. 


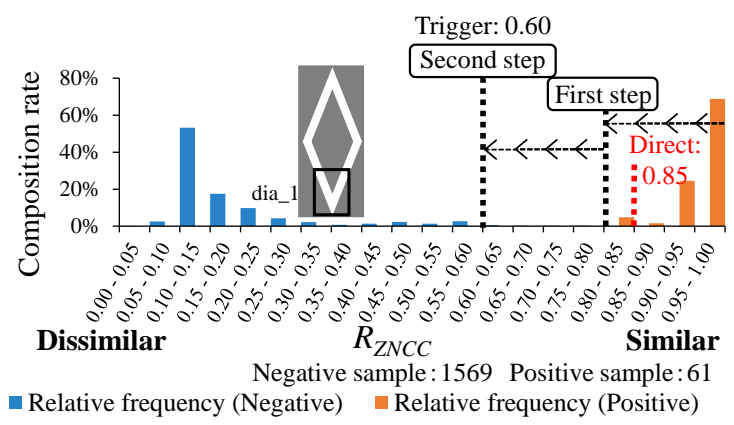

Fig. 14 Histograms of $R_{Z N C C}$ and the threshold value for each role of "dia_1."

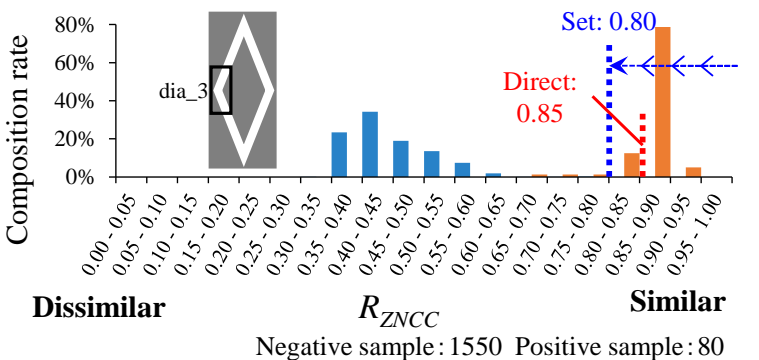

- Relative frequency (Negative) $\quad$ Relative frequency (Positive)

Fig. 15 Histograms of $R_{Z N C C}$ and the threshold value for each role of "dia_3."

Based on the rules discussed above, we determined the thresholds for each partial template of the $40 \mathrm{~km} / \mathrm{h}$ limit marks and the diamarks in accordance with its role. Table 2 shows the threshold for each partial template.

Table 2 Thresholds for each partial template.

\begin{tabular}{|c|c|c|c|c|c|c|c|c|c|c|}
\hline & \multicolumn{9}{|c|}{ Template name } \\
\hline & & 40_1 & 40_2 & $40 \_3$ & $40 \_4$ & $40 \_5$ & dia_1 & dia_2 & dia_3 & dia_4 \\
\hline \multirow{3}{*}{ Role } & Direct & 0.80 & 0.80 & 0.80 & 0.80 & 0.80 & 0.85 & 0.85 & 0.85 & 0.85 \\
\hline & Trigger & 0.55 & & 0.65 & & & 0.60 & 0.65 & 0.65 & \\
\hline & Set & & 0.70 & 0.75 & 0.80 & 0.80 & & 0.80 & 0.80 & 0.85 \\
\hline
\end{tabular}

\subsection{Advantages of Proposed Detection System}

In this section, we summarize the advantages of our proposed system. As we discussed in section 2.2., since the final goal of our system is not the notification of road environments but the localization by detecting road marks, the distance estimation is the important function. Therefore, our system searches the near field using a rear view camera, whose depression angle is large, rather than a front camera, whose depression angle is small. Table 3 shows the comparisons of advantages and disadvantages between the far field detection by the "camera-up system" and the near field detection by the "camera-down system." Although Wu et al. ${ }^{(10)}$ proposed a localization system based on the road mark detection by "camera-up system," they assumed the flat ground or information of the pitch angle of the vehicle. Thus, in order to remove this assumption, they need some other methods, which can cause the error of distance estimation.
Table 3 Comparisons of advantages and disadvantages between "camera-up system" and "camera-down system."

\begin{tabular}{|c|c|c|}
\hline & "Camera-up system" & "Camera-down system" \\
\hline $\begin{array}{l}\text { Conceptual } \\
\text { schematic }\end{array}$ & & \\
\hline Detection field & Far & Near \\
\hline Advantage & $\begin{array}{l}\text { Early detection: The system } \\
\text { can notify the road } \\
\text { environment early because } \\
\text { the camera can see road } \\
\text { marks from a distance. } \\
\text { Whole shape detection: } \\
\text { The camera can take whole } \\
\text { shape of the target road } \\
\text { marks. }\end{array}$ & $\begin{array}{l}\text { Accurate distance estimation: } \\
\text { The system is not susceptible } \\
\text { to postural changes of the } \\
\text { ego-vehicle. } \\
\text { - Robustness against other } \\
\text { vehicles: The coverage field } \\
\text { of the camera is so near that } \\
\text { other vehicles hardly occlude } \\
\text { the target road marks. }\end{array}$ \\
\hline Disadvantage & $\begin{array}{l}\text { - Inaccurate distance } \\
\text { estimation: The system is } \\
\text { susceptible to postural } \\
\text { changes of the ego-vehicle. } \\
\text { - Occlusion by other vehicles: } \\
\text { The target road marks can be } \\
\text { occluded by other vehicle } \\
\text { such as the preceding vehicle. }\end{array}$ & $\begin{array}{l}\text { Late detection: The system } \\
\text { detects road marks only } \\
\text { when the ego-vehicle } \\
\text { approaches the near field or } \\
\text { passes over them. } \\
\text { Partial shape detection: The } \\
\text { camera can only take a part } \\
\text { of the target road marks. }\end{array}$ \\
\hline
\end{tabular}

Regarding the abovementioned "camera-down system," we had to deal with one of its disadvantage: partial shape detection. To solve this problem, as is discussed in section 2.3., our system was designed to obtain the information on types and positions of the target road marks from map in advance. Thus, our system can detect road marks based on the features extracted from the part of road marks which "camera-down system" obtains. Regarding the disadvantage of late detection, there were no actual problems because our system did not aim to notify the road environments.

With respect to the detailed detection algorithms, there are some types of feature extraction method for computer vision such as HOG, Joint-HOG, template matching, Haar-like, and so on. Considering the standardized sizes and shapes of road marks and the robustness against degradation, which affects the performance of edge-based method, we selected the template matching method based on luminance patterns. Furthermore, we applied the ZNCC method that is relatively robust against changes in brightness in order to deal with the influence on road marks by stain, shadow, weather, and so on.

Finally, aiming further detection performance by multiple partial templates, we designed our system to conduct 2 detecting process at the same time: the process based on Direct Template and the process based on the combination of Trigger and Set Template.

Owing to the combinations of the abovementioned elements as a whole system, our proposed system realized road mark detection system based on the "camera-down system" which was not susceptible to postural changes and could estimate accurate distance for localization.

\section{Performance Evaluation Test of Detection Technology of Road Marks on Public Road}

\subsection{Test Conditions}

To evaluate the detection performance of the proposed detection method of road marks based on the partial templates, we conducted the off-line test using the actual driving data on public roads. We set the $10 \mathrm{~km}$ course on a prefectural road, which is not so well-maintained as high-standard roads such as expressways. 
This course contains the $40 \mathrm{~km} / \mathrm{h}$ limit marks at 10 points and the diamarks at 22 points, including some degraded road marks. Fig. 16 shows the examples of degraded road marks on the course.
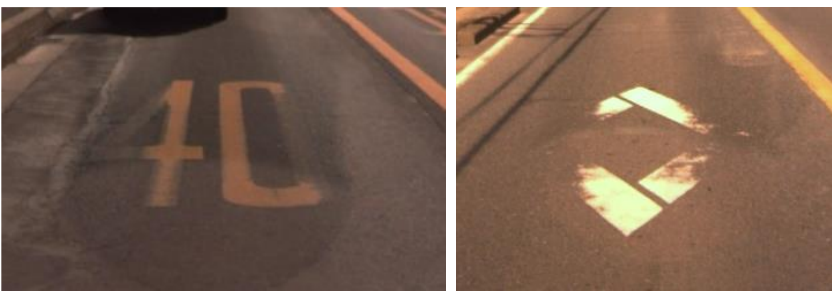

Fig. 16 Examples of degraded road marks on the course.

In addition, to confirm the robustness against the changes in road surface conditions caused by weather changes, we collected the driving data under four weather conditions: sunny conditions on dry roads, cloudy conditions on dry roads, cloudy conditions on half-wet roads, and rainy conditions on wet roads. Moreover, in sunny conditions, since the brightness on the road surface changes in accordance with the time of day, we added the three conditions to the sunny condition: forenoon (from 10 p.m. to 12 p.m.), afternoon (from 2 p.m. to 4 p.m.), and evening (within 2 hours before the sunset). Table 4 shows the example images and corresponding illuminances on each weather condition. The example images consist of the panoramic view and the appearance of the road surface at the fixed point, where we measured the illuminance values by the illuminometer.

Regarding the abovementioned six conditions in total, we selected three driving data for each condition based on the variety of illuminances measured at the start and goal points. However, for the cloudy condition on half-wet roads, we could collect only two driving data.

Table 4 Example images and corresponding illuminances on each weather condition.

\begin{tabular}{|c|c|c|}
\hline $\begin{array}{c}\text { Weather } \\
\text { condition }\end{array}$ & $\begin{array}{c}\text { Sunny } \\
\text { (Dry) }\end{array}$ & $\begin{array}{c}\text { Cloudy } \\
\text { (Dry) }\end{array}$ \\
\hline \multirow{2}{*}{$\begin{array}{c}\text { Example } \\
\text { image }\end{array}$} & &
\end{tabular}

\subsection{Results}

\subsubsection{Processing Speed}

The detection process took 0.03 seconds $(33 \mathrm{~Hz})$ on the average via the desktop PC (3.5 GHz CPU; 8.4 GB memory). Since the framerate of the rear view camera is $30 \mathrm{fps}$, the system realized the real-time detection of road marks. Therefore, the processing speed was practical for localization.

\subsubsection{Detection Results of Road Mark}

Regarding the performance evaluation of the detection method, we defined three results as follows:

- Positive detection: The situation that the system detects at least one region correctly by the Set Templates or the Direct Templates.

- Non-detection: The situation where the system detects no region by Set Templates or Direct Templates although the target road mark exists.

- False detection: The situation that the system detects regions incorrectly by the Set templates or the Direct Templates within the range of $50 \mathrm{~m}$ from the target road mark.

Fig. 17 shows the sequential detection results of the $40 \mathrm{~km} / \mathrm{h}$ limit mark. The orange solid line, green dotted line, and blue dashed line show the RZNCC of "40_1", "40_2", and "40_3", respectively. As is observable in the figure, the $R_{Z N C C}$ values of all the templates exceed the threshold of Direct Template at the appropriate regions.

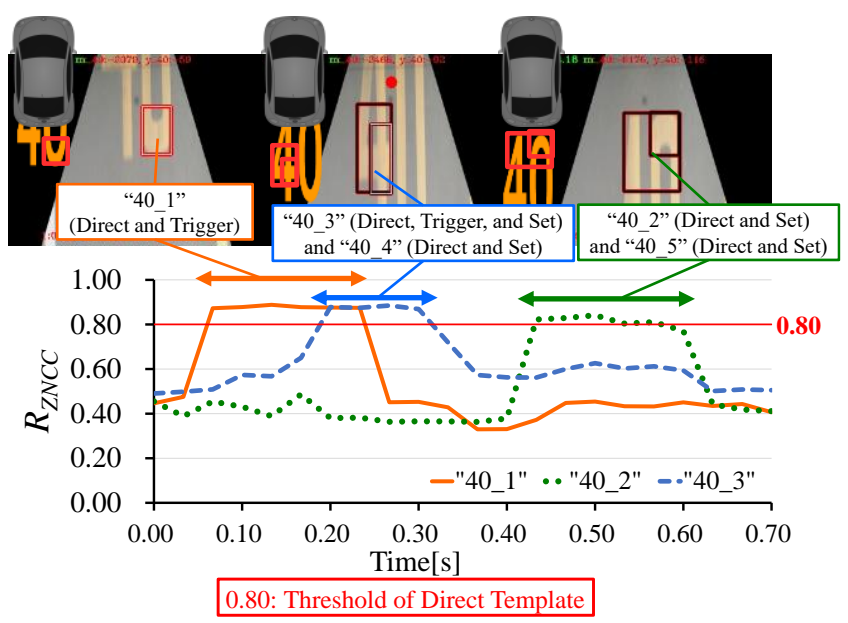

Fig. 17 Sequential detection results of the $40 \mathrm{~km} / \mathrm{h}$ limit mark.

Tables 5 and 6 show the positive detection results of the 40 $\mathrm{km} / \mathrm{h}$ limit marks and the diamarks. As is observable in the tables, the proposed system successfully detected more than $85 \%$ of road marks in any conditions. Moreover, no false detection occurred through the experiment.

Table 5 Positive detection results of the $40 \mathrm{~km} / \mathrm{h}$ limit marks.

\begin{tabular}{|c|c|c|c|c|}
\hline $\begin{array}{c}\text { 40km/h limit } \\
\text { mark }\end{array}$ & $\begin{array}{c}\text { Forenoon } \\
\text { (10 a.m. }-12 \text { p.m. })\end{array}$ & $\begin{array}{c}\text { Afternoon } \\
(2 \text { p.m. }-4 \text { p.m.) }\end{array}$ & $\begin{array}{l}\text { Evening } \\
\text { (Within } 2 \text { hours } \\
\text { before sunset) }\end{array}$ & Total \\
\hline Sunny(Dry) & $87 \%(26 / 30)$ & $97 \%(29 / 30)$ & $100 \%(30 / 30)$ & \multirow{4}{*}{$\begin{array}{c}97 \% \\
(165 / 170)\end{array}$} \\
\hline Cloudy(Dry) & \multicolumn{2}{|c|}{$100 \%(30 / 30)$} & & \\
\hline $\begin{array}{c}\text { Cloudy } \\
\text { (Half wet) }\end{array}$ & \multicolumn{2}{|c|}{$100 \%(20 / 20)$} & & \\
\hline Rainy(Wet) & \multicolumn{2}{|c|}{$100 \%(30 / 30)$} & & \\
\hline
\end{tabular}


Table 6 Positive detection results of the diamarks.

\begin{tabular}{|c|c|c|c|c|}
\hline Diamark & $\begin{array}{c}\text { Forenoon } \\
\text { (10 a.m. }-12 \text { p.m.) }\end{array}$ & $\begin{array}{c}\text { Afternoon } \\
(2 \text { p.m. }-4 \text { p.m. })\end{array}$ & $\begin{array}{l}\text { Evening } \\
\text { (Within } 2 \text { hours } \\
\text { before sunset) }\end{array}$ & Total \\
\hline Sunny(Dry) & $97 \%(64 / 66)$ & $98 \%(65 / 66)$ & $100 \%(66 / 66)$ & \multirow{4}{*}{$\begin{array}{c}99 \% \\
(371 / 374)\end{array}$} \\
\hline Cloudy(Dry) & \multicolumn{2}{|c|}{$100 \%(66 / 66)$} & & \\
\hline $\begin{array}{c}\text { Cloudy } \\
\text { (Half wet) }\end{array}$ & \multicolumn{2}{|c|}{$100 \%(44 / 44)$} & & \\
\hline Rainy(Wet) & \multicolumn{2}{|c|}{$100 \%(66 / 66)$} & & \\
\hline
\end{tabular}

Figs. 18 and 19 show the examples of detected road marks affected by disturbances. As is shown in Fig. 18, since the center regions of the diamark are covered with shadows, the $R_{Z N C C}$ values of "dia_2" and "dia_3" do not exceed the thresholds of either Direct Template or Set Template. However, the system detects the road mark based on the detection result of "dia_1" and "dia_4", which are not covered with shadows. In other words, Fig. 18 shows the successful example of focusing on characteristic regions. On the other hand, as is observable in the Fig. 19, since the both side regions of the $40 \mathrm{~km} / \mathrm{h}$ limit mark are degraded, all the RZNCC values do not exceed 0.80 , which is the threshold of Direct Template. However, the proposed system successfully detects it based on the combination of "40_1" (Trigger Template) and "40_3" (Set Template). In other words, Fig. 19 shows the successful example of the combination of the multiple templates.

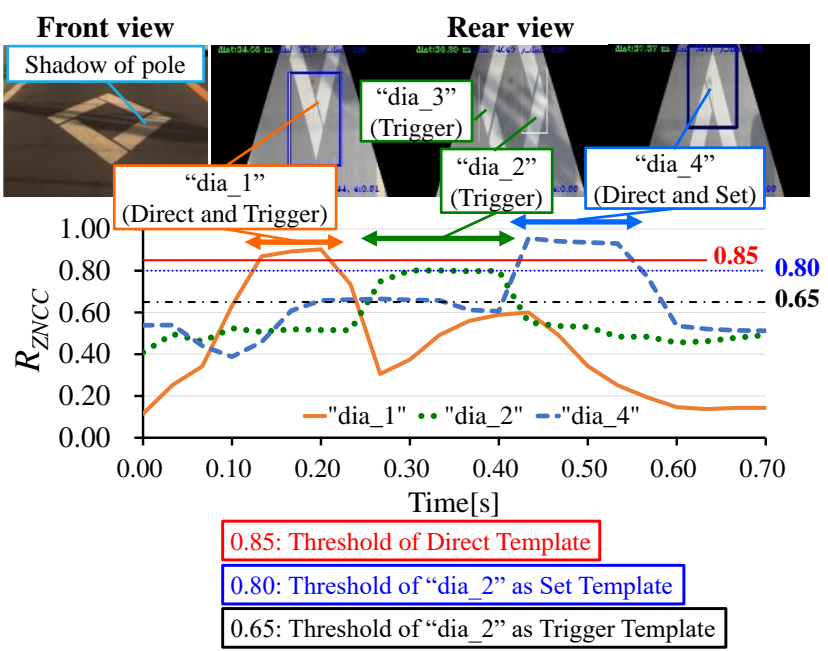

Fig. 18 Example of detected road marks partly covered with shadows.

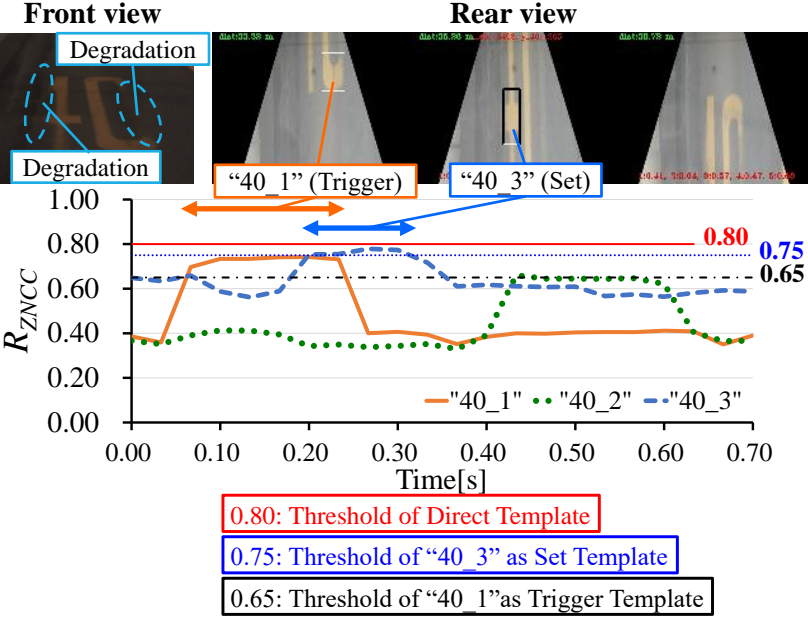

Fig. 19 Example of detected road marks with degradation.

Fig. 20 shows the examples of detected road marks in wet conditions. In wet and half-wet conditions, the color of road surface is darker than in dry conditions. Thus, the luminance patterns of road marks are easy to extract from the surrounding regions, and the system detects road marks without any problems.

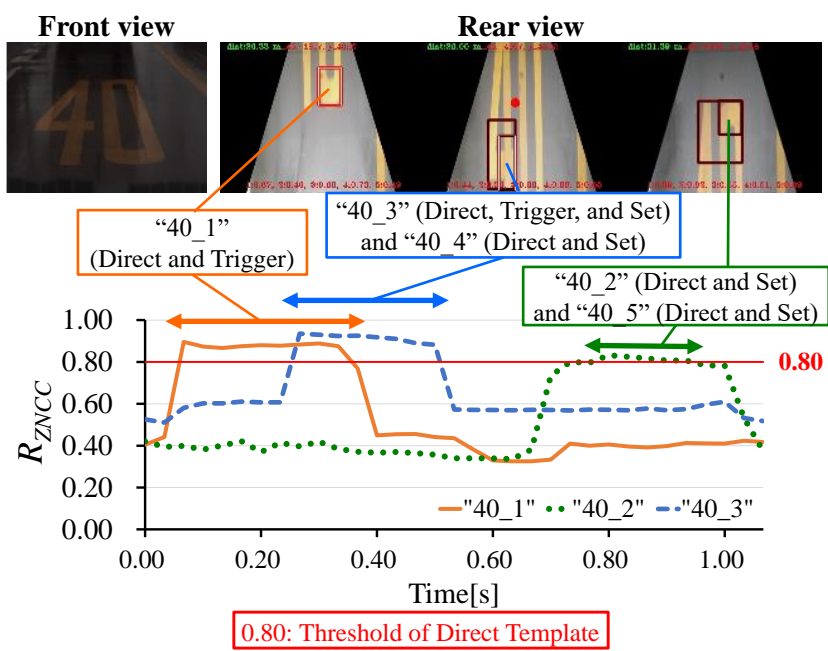

Fig. 20 Example of detected road marks in wet conditions.

However, in the forenoon and the afternoon on sunny days, our system could not detect five $40 \mathrm{~km} / \mathrm{h}$ limit marks and three diamarks. Figs. 21 and 22 show the examples of non-detected road marks. As is observable in the Fig. 21, although the regions corresponding to "dia_1" and "dia_4" are not degraded unlike those corresponding to "dia_2" and "dia_3", the shadows covering these regions prevent them from being detected. 


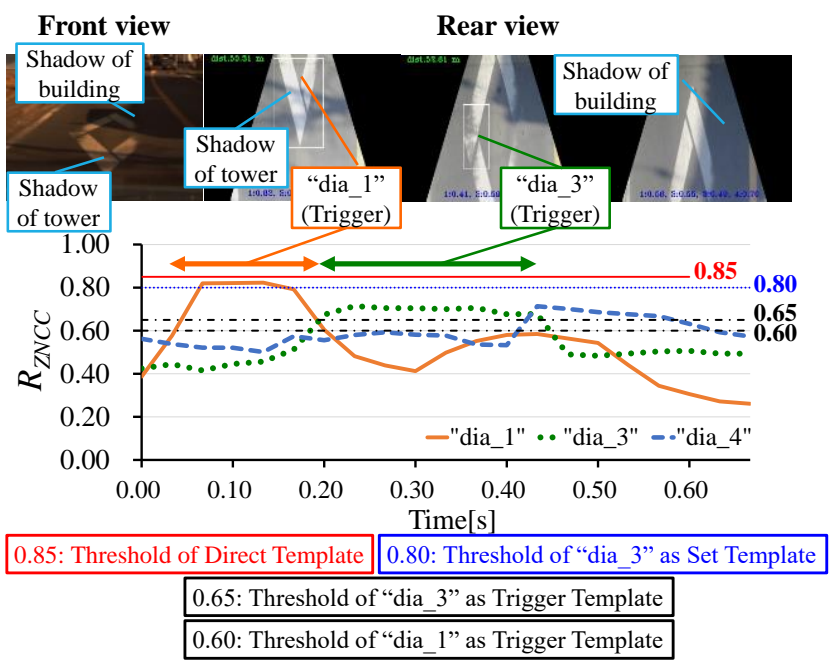

Fig. 21 Example of non-detected road marks covered with shadow.

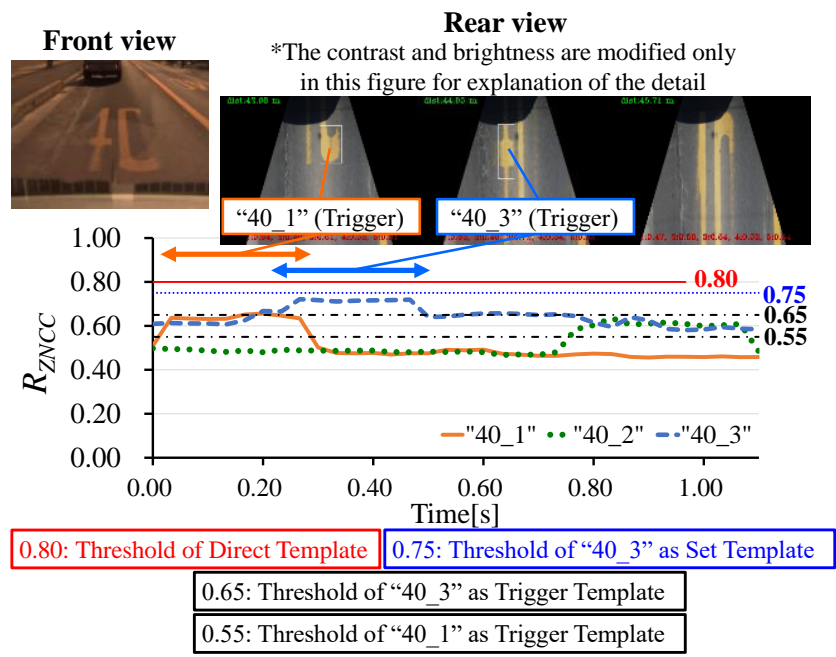

Fig. 22 Example of non-detected road marks in sunny conditions.

On the other hand, as is shown in the Fig. 22, although the regions corresponding to "40_1", "40_2", and "40_3" are not so degraded, high brightness of the road surface affects the luminance patterns of these regions. In fact, the mean value of measured illuminances in the forenoon and the afternoon on sunny days was approximately 75,000 lx, which was more than three times higher than other conditions. Regarding this type of problem caused by brightness, thresholds determined for each weather conditions have the possibility of solving the problem. In addition, considering the recent popularization of cameras for ADAS, wide dynamic-range cameras also can solve the problem.

In conclusion, in spite of disturbances, the proposed system successfully detected 536 of road marks on the public road, whose total distance is approximately $170 \mathrm{~km}$. Based on a simple calculation, we can realize localization every $300 \mathrm{~m}$; thus, we confirmed that our system has practical performances for detecting the road marks as the references of localization on the actual public roads.

\section{Conclusions}

To realize the intelligence for automated vehicles, we aimed to develop the accurate localization system using the landmarks. To this end, we selected the road marks as the landmarks and proposed the detection method robust against the disturbances based on the combinations of characteristic regions of road marks; further we implemented the prototype of proposed technology. In addition, the performance evaluation test using driving data on public roads showed that the proposed system can detect road marks robustly and has the possibility of realizing frequent localization on public roads.

As future works, we need to implement further types of road marks as landmarks such as the $30 \mathrm{~km} / \mathrm{h}$ limit marks, the $50 \mathrm{~km} / \mathrm{h}$ limit marks, and so on. In addition, we need to discuss the system which actively changes the threshold values for detection in accordance with the weather conditions. Furthermore, we need to develop the technology that calculates the accurate position on map based on the detection result of road marks.

This paper is written based on a proceeding presented at JSAE FAST-zero'17 Meeting.

\section{Acknowledgement}

This research has been conducted as a part of the research project "Autonomous Driving System to Enhance Safe and Secured Traffic Society for Elderly Drivers" granted by Japan Science and Technology Agency(JST), Strategic Promotion of Innovative Research and Development.

\section{References}

(1) Sebastien Glaser, Lydie Nouveliere, and Benoit Lusetti: Speed Limitation Based on an Advanced Curve Warning System: 2007 IEEE Intelligent Vehicles Symposium, pp.686691(2007).

(2) Jeremie Daniel, Gaétan Pouly, Abderazik Birouche, and Michel Basset: Navigation-based speed profile generation for an open road speed assistant: Proceedings of the 12th IFAC Symposium on Transportation Systems, Vol. 42, Issue 15, pp.320-327(2009).

(3) Keisuke Yoneda, Hossein Tehrani, Takashi Ogawa, Naohisa Hukuyama, and Seiichi Mita: Lidar Scan Feature for Localization with Highly Precise 3-D Map: 2014 IEEE Intelligent Vehicles Symposium Proceedings, pp.1345-1350 (2014).

(4) Liang Wang, Yihuan Zhang, and Jun Wang: Map-Based Localization Method for Autonomous Vehicles Using 3DLIDAR: IFAC-PapersOnLine, Vol. 50, Issue 1, pp.276281(2017).

(5) Shinpei Kato, Eijiro Takeuchi, Yoshio Ishiguro, Yoshiki Ninomiya, Kazuya Takeda, and Tsuyoshi Hamada: An Open Approach to Autonomous Vehicles: IEEE Micro, Vol. 35, Issue 6, pp.60-68(2015).

(6) Takuma Ito, Masahiro Mio, Kyoichi Tohriyama, and Minoru Kamata: Novel Map Platform based on Primitive Elements of Traffic Environments for Automated Driving Technologies: 
International Journal of Automotive Engineering 7, pp.143151 (2016).

(7) Masafumi Noda, Tomokazu Takahashi, Daisuke Deguchi, Ichiro Ide, Hiroshi Murase, Yoshiko Kojima, and Takashi Naito: Recognition of Road Markings from In-Vehicle Camera Images by a Generative Learning Method: Proceedings of the IAPR Conference on Machine Vision Applications, pp.514-517 (2009).

(8) Shogo Yoneyama, Takashi Hirano, Kageyasu Miyahara, and Takenori Kawamata: Recognition of Road Marking Using HMM: IEICE technical report, Vol. 109, No. 182, pp.13-18 (2009) [published in Japanese].

(9) Hideyuki Ishida, Kiyosumi Kidono, Yoshiko Kojima, and Takashi Naito: Road marking recognition for map generation using sparse tensor voting: Proceedings of 21st International Conference on Pattern Recognition, pp.1132-1135 (2012).

(10) Tao $\mathrm{Wu}$ and Ananth Ranganathan: Vehicle Localization using Road Markings: Proceedings of 2013 IEEE Intelligent Vehicles Symposium (IV), pp.1185-1190 (2013). 